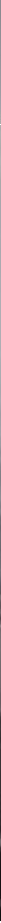

\title{
O Planejamento Estratégico como condicionante da Elaboração Orçamentária
}

\section{Flávio Sergio Rezende Nunes de Souza}

Capitão-de-Corveta (IM). Encarregado da Seção de Planejamento Estratégico da Diretoria de Administração da Marinha. Mestre em Administração Pública (EBAPE/FGV). E-mail: flavio.nunes@dadm.mar.mil.br

\section{Thiago José Parreira}

Capitão-de-Corveta (IM). Chefe do Departamento de Alunos do Centro de Instrução e Adestramento Almirante Newton Braga. Mestre em Administração Pública (EBAPE/FGV). E-mail: parreira@cianb.mar.mil.br

\section{Carolina Meira Dyott Fontenelle}

Advogada. Especialista em Direito Público (Universidade Estácio de Sá). E-mail: carolinafontenelle.adv@gmail.com

\section{RESUMO}

Para possibilitar o atendimento de demandas crescentes, e a cada dia mais complexas da sociedade, a Administração Pública necessita utilizar ferramentas que permitam a condução de suas unidades a determinadas direções, buscando-se a melhoria dos serviços públicos e a redução do desperdício. Nesse cenário, o planejamento estratégico se mostra imprescindível, porém não basta que esse seja formulado, deve ser efetivamente implementado, permeando os demais processos organizacionais. Este ensaio tem a finalidade de promover uma

\section{ABSTRACT}

In order to address demands of a growing and increasingly complex society, public organizations need to use tools that allow their units being driven to certain directions, seeking the improvement of public services and waste reduction. In this scenario the strategic planning proves being essential. Formulation is not enough but must be effectively implemented, thus permeating the other processes of the organization. Hence, this essay intends to promote discussion on the implementation of strategic planning in the public sector, addressing its 
discussão sobre a implementação do planejamento estratégico no setor público, abordando seu relacionamento com os sistemas gerenciais, e em especial o sistema orçamentário, que possui extrema relevância nesse setor. Para tal, procura identificar como o planejamento estratégico, condiciona a elaboração orçamentária nas unidades, promovendo um debate sobre os benefícios desta integração, assim como sobre os obstáculos que podem inviabilizá-la.

Palavras-chave: Planejamento estratégico; Setor Público; Elaboração Orçamentária. relationship with management systems, and in particular with budgetary system which is vital to this sector. Furthermore, the report aims to identify how the strategic planning are linked to budget preparation, also promoting a debate about the benefits of this integration as well as the obstacles that can make it impracticable.

Keywords: Strategic planning; Public Sector; Budget preparation.

\section{INTRODUÇÃO}

Apesar do desenvolvimento de diversas técnicas empregadas na gestão estratégica, a implementação das estratégias continua sendo uma etapa que apresenta grande dificuldade. Muitas vezes, as organizações possuem planos muito bem elaborados, mas as suas ações efetivamente não se alinham a estes planos, que se tornam apenas documentos sem um propósito maior, que não servem como guias para a melhoria da gestão nessas organizações.

Diversos autores afirmam que muito já foi escrito sobre estratégia e planejamento estratégico no setor privado, porém que o setor público ainda é pouco explorado (BERRY; WECHSLER, 1995; JOYCE, 1999; ROSE; CRAY, 2010; STEWART, 2004). Em parte, isto se deve à inserção da área de estratégia na Administração Pública ser relativamente nova e de pouca expressão (FERREIRA; NAJBERG; SOUSA, 2013; LLEWELLYN; TAPPIN, 2003).

No Brasil, Bertero, Vasconcelos e Binder (2003) ao pesquisarem a produção científica em estratégia no período entre 1991 e 2002, encontraram apenas seis trabalhos publicados sobre estratégia no setor público, o que correspondia a apenas $2 \%$ de todos os trabalhos publicados nesta temática. Mais recentemente, Ferreira, Najberg e Sousa (2013) realizaram pesquisa bibliométrica em periódicos na àrea de administração com classificação qualis de A1 a B3, utilizando a base Scielo Brasil, no período entre 2007 a 2012. Foram encontrados apenas 25 artigos sobre estratégia no setor público, sendo que apenas 6 tratavam sobre planejamento estratégico. Devido ao pequeno número de trabalhos encontrados, os autores sugerem a realização de mais pesquisas nesta área.

Apesar da maior parte das pesquisas versarem sobre formulação de estratégias, a própria literatura aponta a importância da implementação. Para Eadie (1983) a experiência indica que o sucesso na adoção do planejamento estratégico depende de se dar mais atenção à implementação das estratégias do que às abordagens analíticas de formulação de estratégias. Tem sido cada vez mais reconhecido que essa etapa é um grande desafio para líderes estratégicos e gestores de serviços públicos (FLYNN; TALBOT, 1996; JOYCE, 1999).

Em um nível mais específico, alguns autores apontam a existência de poucas pesquisas examinando como sistemas gerenciais são utilizados em organizações para facilitar ou apoiar mudanças estratégicas (ABERNETHY; BROWNELL, 1999), e quais as consequências deste uso (SHIELDS, 1997).

Para Abernethy (1999), muitos pesquisadores ignoram o potencial da utilização dos sistemas gerenciais como ferramentas mais ativas na formulação e implementação de mudanças na direção estratégica, o que Simons (1994) denomina de uso interativo destes sistemas.

Especialmente em relação aos sistemas orçamentários, Shields (1997) identificou que quase todas as pesquisas são basicamente de dois tipos: o primeiro fundamenta-se na teoria da agência, tratando de estudos analíticos que derivam desenhos de sistemas orçamentários buscando maximizar retornos esperados; e o segundo tipo investiga características dos efeitos do orçamento relacionadas com comunicação, atitudes e desempenho. Esse autor sugere, entre outras temáticas, a realização de pesquisas relacionadas a processos estratégicos, e como esses se integram com o orçamento.

Assim, teoricamente, este ensaio contribui de forma a explorar a escassez de pesquisas relativas à implementação do planejamento estratégico na Administração Públi- 
$\mathrm{ca}$, e ao seu relacionamento com os sistemas gerenciais, e em especial o sistema orçamentário, que possui extrema relevância nesse setor. Para tal, procura identificar como as ações que deveriam ser condicionadas pelo planejamento estratégico se vinculam a ele, apontando facilitadores e obstáculos dessa relação.

\section{PLANEJAMENTO ESTRATÉGICO}

Mintzberg e Quinn (2001) afirmam que a estratégia define uma base contínua para ordenar as adaptações da empresa (nas suas táticas) a fim de direcioná-las a propósitos concebidos de forma mais ampla.

Em relação ao termo planejamento, Mintzberg (2004) identifica diversas definições, que vão desde as mais amplas, sendo desta forma muito genéricas, até as mais estritas, que na opinião do autor, seriam as mais adequadas. Assim, pode-se afirmar que "planejamento é um procedimento formal para produzir um resultado articulado, na forma de um sistema integrado de decisões" (Mintzberg, 2004, p.26). Neste conceito, destaca-se a formalização, que é formada por três componentes: decompor; racionalizar os processos; e articular. Enquanto que os dois primeiros permitem que os planos sejam decompostos de modo lógico e realista em estratégias, programas, orçamentos e objetivos, a articulação torna as coisas explícitas, com a finalidade de formar um sistema integrado de decisões (MINTZBERG, 2004; PEARCE; FREEMAN; ROBINSON, 1987).

Para Anthony (1965, p.16), planejamento estratégico é "o processo de decidir os objetivos da organização, as mudanças destes objetivos, os recursos utilizados para alcançar estes objetivos, e as políticas que direcionam a aquisição, uso e disposição destes recursos". Desta forma, este combina duas ações: escolher os objetivos e planejar como alcançá-los.

Este autor estabelece um modelo em que o planejamento estratégico se relaciona intimamente com o controle gerencial, que é o processo pelo qual os gerentes se certificam que os recursos são obtidos e utilizados efetivamente e eficientemente no cumprimento dos objetivos organizacionais.

Na década de 90, a formulação e a implementação das estratégias passam a ser integradas, eliminando a separação entre aqueles que pensam e planejam e aqueles que executam as ações na organização. Tal visão vem a consolidar a chamada Gestão Estratégica (BERTERO; VASCONCELOS; BINDER, 2003).

Desta forma, percebe-se que não basta um planejamento estratégico bem elaborado, é necessário que haja uma relação adequada com o controle gerencial, devendo este último, funcionar bem, para que seja possível o alcance dos objetivos estabelecidos.

\section{GESTÃO ESTRATÉGICA NA ADMINISTRA- ÇÃO PÚBLICA}

O crescimento e a complexidade de algumas organizações públicas, aliados ao ambiente conturbado no qual estão inseridas, tornam a gestão estratégica indispensável. Estas são pressionadas por diversas expectativas, necessitando utilizar instrumentos que as conduzam a determinadas direções, na busca da melhoria dos resultados e da eficiência dos serviços públicos (JOYCE, 1999).

Joyce (1999) identifica quatro modelos de gestão estratégica na Administração Pública: o modelo clássico de planejamento; o modelo similar ao empresarial; o modelo visionário; e o modelo baseado em previsão.

No primeiro, a abordagem constitui-se em um endosso de um planejamento racional direcionado à gestão. Para muitas organizações públicas a formulação deste planejamento ocorre de maneira imposta, não sendo uma escoIha da organização (FLYNN; TALBOT, 1996; LLEWELLYN; TAPPIN, 2003). Porém, tal medida pode levar a criação de muitos planos estratégicos formalmente escritos, sem a garantia de que expressarão algum pensamento estratégico. Estes podem até satisfazer em alguns casos os órgãos de supervisão administrativa, porém, por outro lado estarem sendo inúteis em termos gerenciais.

Essa lógica de planejamento estratégico sofreu uma reviravolta na década de 90 , ganhando maior visibilidade em sua importância. $O$ argumento de que o setor público estava falhando no atendimento das demandas da população teria como solução a adoção de uma forma de governo mais empreendedora. Essa envolvia diversas medidas como o uso de competição, a flexibilidade de gestão, o foco na qualidade dos serviços, a delegação de autoridade, além do planejamento estratégico, ferramenta esta que permitiria uma postura proativa na gestão pública (OSBORNE; GAEBLER, 1996). 
Essa ferramenta, ao antecipar situações futuras, torna possível o uso de medidas preventivas, que supostamente são mais econômicas. Por isso, sua utilização pelos modernos serviços públicos para combater problemas de recursos insuficientes passou a ser influente (JOYCE, 1999).

Em pesquisa realizada em governos locais do Reino Unido, Flynn e Talbot (1996) verificaram que a maioria das organizações tinha planejamentos estratégicos formais, e que isto levava a diversos benefícios. Na percepção dos gestores dessas organizações, esses planos os auxiliavam a atingir metas e objetivos, a identificar marcos para melhorias organizacionais e a melhorar o uso dos recursos. Além disso, auxiliava a criar unicidade na visão futura da organização por parte dos funcionários, favorecendo a identificação de novas oportunidades e ideias.

A base do segundo modelo está no tratamento dos usuários dos serviços públicos de maneira melhor. Assim, estes deveriam ser tratados como "clientes". Esta visão passou a ser aplicada inclusive nas relações internas das organizações, aplicando-se uma abordagem cliente-fornecedor. Uma característica fundamental deste modelo é o desenvolvimento de um núcleo estratégico dentro da organização pública ao mesmo tempo em que o poder é devolvido aos gestores que se encontram na linha de frente. Entretanto, à medida que estes gestores recebem maior poder, também se tornam mais responsáveis pelo desempenho de suas unidades.

O modelo de gestão estratégica visionária se preocupa mais com o longo prazo. Em sua essência, é definido um futuro desejado para determinado serviço público e, então, identificadas as atividades que levarão a organização a esse estado desejado. Enquanto esta visão é definida pela alta administração, os gestores da organização se preocupam com o processo de internalização dos valores que promoverão esta visão.

Finalmente, o modelo baseado em previsão é fundamentado na ideia de orientação da ação estratégica pelo uso de inteligência. Este implica em imaginar um futuro, que projeta o que é desejável no presente, e então, planejar um caminho para este futuro. Este desenvolve uma visão estratégica, assim como o modelo anterior, porém nesse caso a simples identificação das atividades não possibilita a organização alcançar esse futuro. A organização se concentra no desenvolvimento de áreas especiais de conhecimento, e de empreendimentos coopera- tivos com outras organizações, a fim de garantir o alcance de sua visão estratégica.

\section{IMPLEMENTAÇÃO DO PLANEJAMENTO ES- TRATÉGICO}

O planejamento estratégico deve ser implementado através de planos de ação, que possuem um horizonte de tempo mais curto e que devem estar alinhados com os objetivos e diretrizes daquele (LOBATO, 2000). As ações contidas nesses planos contribuirão na conquista dos resultados desejados, contidos no planejamento maior:

(...) a estratégia se torna real quando uma organização se assegura que esta foi implementada. Internamente, isto significa que a estratégia é utilizada para planejar e controlar comportamentos. Objetivos operacionaise metas de desempenho são definidos com base na estratégia (Joyce, 1999, p.3).

Muitas vezes os planos estratégicos não passam de documentos formais, com um conteúdo que não reflete a realidade. Esse caso é notório no setor público, onde as organizações frequentemente realizam sua formulação por exigências estatutárias, ou seja, o que leva à sua criação não são as escolhas estratégicas (FLYNN; TALBOT, 1996; LLEWELLYN; TAPPIN, 2003).

Nesta perspectiva, este planejamento tem sua importância esvaziada. Apesar de serem formulados ficam praticamente sem uso. Tal fenômeno foi denominado por Llewellyn e Tappin (2003, p.968) de documentos dormentes: "planos estratégicos residiam nas prateleiras, juntando poeira. Essa documentação dormente é um fenômeno transatlântico".

A implementação requer alguns passos a serem executados de forma racional, como: o desenho de planos de ação; a designação individualizada de responsabilidades específicas; o cálculo dos custos de implementação de novas ações, assegurando que estes foram incluídos no processo orçamentário anual; e o estabelecimento de sistemas de informações e monitoramento, para assegurar o cumprimento de metas, assim como, eventuais correções nos sistemas de controle. Além disso, elementos mais subjetivos também são imprescindíveis, como o papel da liderança, que tem a finalidade de conquistar o apoio dos stakeholders no processo de implementação (JOYCE, 1999). 
Assim, verifica-se a importância do alinhamento do planejamento estratégico com outros processos ou subsistemas, assegurando a unidade de propósito e integração para todos os níveis gerenciais que estarão envolvidos (JOYCE, 1999; MIESING, 1984).

Em muitas organizações públicas não existe um sistema de planejamento integrado e que englobe diversos níveis organizacionais. Para Stewart (2004), a utilização da gestão estratégica no sentido de relacionar sistemas decisórios com objetivos de longo prazo no setor público é uma raridade.

Apesar disso, ao longo do tempo espera-se que haja nas organizações do setor público uma maior integração do planejamento com os níveis operacionais (BERRY; WECHSLER, 1995).

Além disso, pode haver problemas relacionados ao tipo de sistema de planejamento implantado. Nutt e Backoff (1993) identificaram problemas relacionados a múltiplos centros estratégicos, a que chamaram de "empowered organization". Neste tipo de organização a estratégia era criada simultaneamente em todos os níveis, levando a problemas de integração e comunicação.

Uma barreira clássica para a implementação do planejamento estratégico, é a falta de envolvimento da alta administração. Os dirigentes devem ter de forma bem clara a ideologia central, os valores e objetivos da organização, e com base nesses respaldar a colocação em prática dos planos de ação (FLYNN; TALBOT, 1996; LOBATO, 2000).

Além disso, a literatura aponta outros problemas na implementação relacionados à incapacidade de comunicação e preparação das mudanças. Ainda, no caso do Brasil, existem obstáculos associados a questões culturais, como a improvisação, o imediatismo e o individualismo do brasileiro (LOBATO, 2000).

No caso de estratégias que requeiram maiores mudanças, um grande obstáculo é a repressão da incerteza. Esta ocorre devido à busca de segurança e regularidade na vida empresarial. Desta forma, o gestor tenta contornar a pressão psicológica de ter que decidir com base em fatores que fogem de seu controle (MOTTA, 2002).

Dentre as formas de repressão ressaltam-se: o planejamento por mini análise; a definição de objetivos amplos e imprecisos; a dissociação planejamento-execução; além do incrementalismo e a reconciliação com o passado.

Na primeira forma dificultam-se os debates e as análises de metas, de forma a se garantir o ponto de vista dos dirigentes. Tal prática leva a acomodações nos outros níveis. Apesar dos técnicos da organização possuírem informações que indiquem a necessidade de mudança, esses passam a evitar a colocação desses assuntos em pauta, com a percepção de que isto será inútil.

Na segunda forma, são estabelecidos objetivos que sejam vagos e pouco desafiadores, assim produz-se a falsa impressão de que sempre estão sendo alcançados.

$\mathrm{Na}$ dissociação planejamento-execução costuma-se isolar a decisão da ação. Essas fases têm que atuar conjuntamente, caso contrário todo esforço estratégico já realizado terá sido em vão.

Por último, no incrementalismo "existe a tendência de apegar-se ao conhecido e experimentado, tomando-se decisões estratégicas exclusivamente em bases incrementalistas" (Motta, 2002, p.96). Assim, antes de se buscar mudanças que façam a organização evoluir com base em uma nova visão de futuro, sempre se olha para trás para ver o que vem sendo feito, desta forma, evitam-se grandes alterações no status quo.

\section{4.1 A Integração entre Orçamento Público e o Planejamento Estratégico}

Na Administração Pública, o orçamento é um importante instrumento para a implementação da visão estratégica das organizações nos níveis táticos e operacionais:

$O$ desejo de mover os serviços públicos de uma cultura burocrática e complacente para outra mais empreendedora é também fonte de um desejo de estreitar a relação entre planejamento estratégico e sistemas orçamentários. Defensores do governo empreendedor atualmente querem inter-relacionar declarações de missão, com os sistemas orçamentários e com medidas de desempenho e gestão. (...) consequentemente, querem ver os executores do orçamento focados na tradução dos orçamentos em determinados níveis de desempenho, que são estabelecidos com base na declaração de missão e nos objetivos estratégicos gerais, formulados dentro do processo de planejamento estratégico (Joyce, 1999, p. 73).

Por outro lado, pode-se verificar que a relação entre o planejamento estratégico e o sistema orçamentário também ocorre no sentido contrário, o que faz com que esta 
seja ainda mais relevante. Ou seja, o planejamento não só condiciona a alocação orçamentária, assim como necessita dos recursos orçamentários para que seja efetivamente implementado. Desta forma, deve-se assegurar que o planejamento estratégico influencie o processo de alocação orçamentária (BERRY; WECHSLER, 1995; EADIE, 1983; GOODWIN; KLOOT, 1996; JOYCE, 1999).

Para Cunha (1999), atualmente há uma visão limitada em relação ao papel do orçamento público como instrumento de gestão. 0 que predomina é uma visão legalista, na qual se vê o orçamento como um instrumento apenas de legitimação do gasto público.

As diversas atividades orçamentárias encontram-se, na verdade, nos três níveis - planejamento estratégico, controle gerencial, e controle operacional - sendo essenciais para o funcionamento da organização (ANTHONY, 1965; CUNHA, 1999; SCHICK, 1966), conforme ilustrado na Figura 1 .

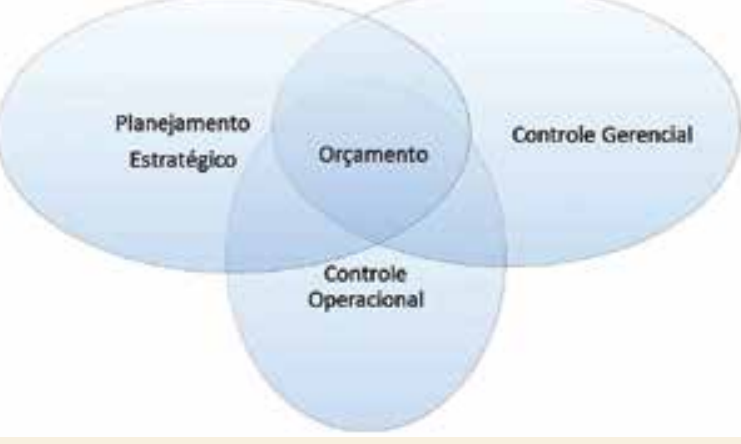

- Figura 1: 0 orçamento e os processos administrativos

Fonte: Adaptado de Cunha (1999), Schick (1966) e Anthony (1965).

Se o planejamento estratégico não estiver integrado com o processo orçamentário anual, assim como com outros importantes sistemas de gestão, a implementação da estratégia não será efetiva, desta forma, não havendo como se falar em gestão estratégica (JOYCE, 1999).

Goodwin e Kloot (1996) encontraram estreita integração entre os processos orçamentários e o planejamento estratégico em administrações locais da Nova Zelândia, enquanto que na Austrália encontraram ligações vagas ou inexistentes. No primeiro país as estratégias eram revisadas, levando a mudanças em seus planos estratégicos, e posteriormente, os orçamentos para o exercício financeiro seguinte eram decididos. Por outro lado, na Austrália o processo decisório era de natureza incremental.

No primeiro caso, uma estreita ligação entre os processos leva a um alto grau de incerteza aos gestores intermediários, tendo em vista que estes sabem que terão que dar atenção não só para o processo orçamentário, como também ao planejamento estratégico. Assim, segundo estes autores, o que assegura uma menor ambiguidade no papel destes gestores, reduzindo a incerteza, é uma boa comunicação estratégica, que permitirá que a informação seja difundida para os níveis inferiores.

Outra experiência prática que possibilitou o rompimento com o incrementalismo, além da integração entre orçamento e estratégia, foi o caso do programa "Brasil em Ação".Nesse caso, foi possivel implementar uma estrutura de projetos, executados de forma transversal entre os ministérios, que possibilitou levar adiante programas de infraestrutura e sociais, de caráter estratégico do governo. Do mesmo modo, evitou que os ministérios isoladamente tomassem decisões orçamentárias, o que poderia inviabilizar os objetivos estratégicos do governo (BARZELAY; SHVETS, 2005).

\section{- 4.2 Modelo conceitual}

Do modelo de Anthony (1965), discutido por Schick (1966) e Cunha (1999), enfocando partes específicas do planejamento estratégico (ações estratégicas que têm impacto orçamentário) e da elaboração da proposta orçamentária nas organizações, deriva o esquema constante da Figura 2, que demonstra a relação entre essas variáveis.

O planejamento estratégico estabelece o sentido de direção a ser seguida pela organização para cumprir a missão institucional. Para alcançar os objetivos, são estabelecidas ações estratégicas a serem executadas pelos diversos sistemas organizacionais. A coordenação subjacente a essa relação consiste em uma das próprias finalidades do planejamento estratégico (MINTZBERG, 2004; VILÀ; CANALES, 2008).

Grande parte do planejamento estratégico implica em ações que têm impacto orçamentário. Essas devem condicionar as propostas orçamentárias posteriores à entrada em vigor dos planos estratégicos em que estão inseridas, de forma que os recursos necessários para a execução dessas sejam solicitados. Não obstante, deve-se considerar a influência de alguns fatores que podem servir como catalisadores desse vínculo, ou dificultá-lo. 
$<<$

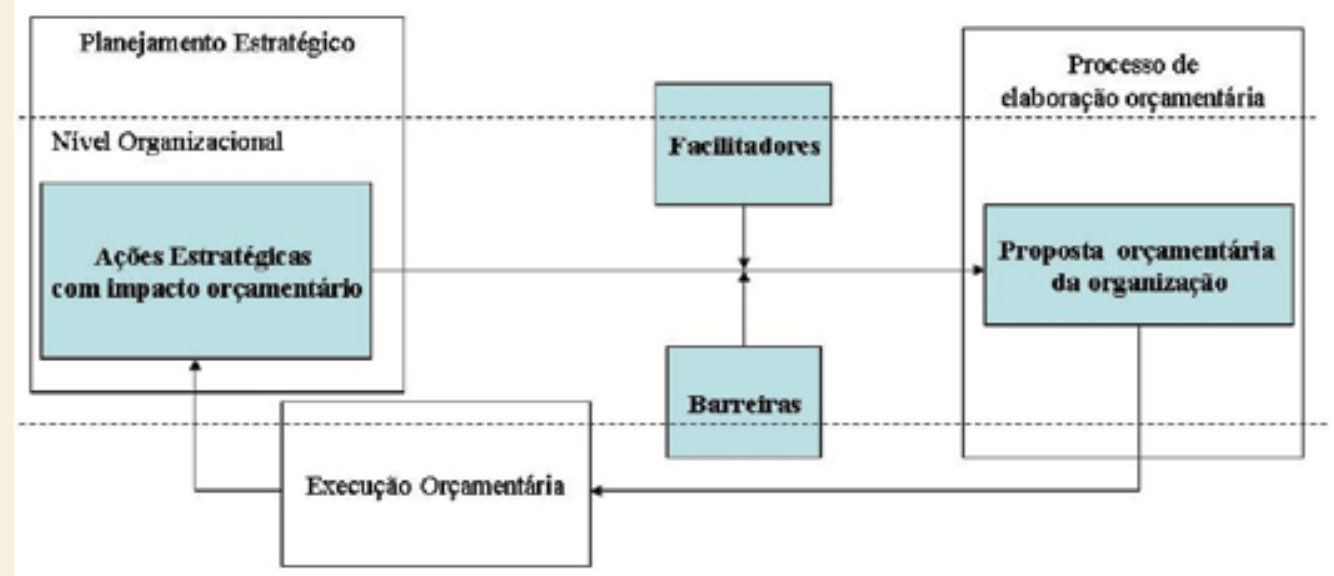

- Figura 2: Relação entre ações estratégicas que possuem impacto orçamentário e a elaboração da proposta orçamentária nas organizações

$\square$ Fonte: Elaborado pelos autores.

Algumas perspectivas da análise organizacional estabelecidas por Motta $(1998$, p.73) foram utilizadas para organizar os fatores (facilitadores ou barreiras) encontra- dos na literatura que interferem na relação estabelecida no modelo apresentado. A classificação desses fatores encontra-se representada no Quadro 1.

Quadro 1: Fatores que influenciam a relação entre as ações estratégicas com impacto orçamentário e a elaboração das propostas orçamentárias

\begin{tabular}{|c|c|}
\hline PERSPECTIVA & Fatores \\
\hline \multirow{5}{*}{ ESTRUTURAL } & Incapacidade de comunicação e preparação das mudanças (LOBATO, 2000; VILÀ; CANALES, 2008) \\
\hline & Designação individualizada de responsabilidades (JOYCE, 1999) \\
\hline & Ambiguidade no papel orçamentário (GOODWIN; KLOOT, 1996) \\
\hline & Falta de sistema de planejamento integrado com níveis inferiores (FLYNN; TALBOT, 1996; STEWART, 2004) \\
\hline & Dissociação planejamento-execução (MOTTA, 2002) \\
\hline \multirow{6}{*}{ TECNOLÓGICA } & Incrementalismo e reconciliação com o passado (MOTTA, 2002) \\
\hline & $\begin{array}{l}\text { Imposição externa para a formulação (FLYNN; TALBOT, 1996; JOYCE, 1999); "documentação dormente" } \\
\text { (LLEWELLYN; TAPPIN, 2003) }\end{array}$ \\
\hline & $\begin{array}{l}\text { Formulação integrada à implementação (BERTERO; VASCONCELOS; BINDER, 2003; MOTTA, 2002); elabo- } \\
\text { ração de plano de ação (LOBATO, 2000) }\end{array}$ \\
\hline & Orçamentação dupla (JOYCE, 1999) \\
\hline & Problemas no tipo de sistema de planejamento implantado - empowered organization (NUTT;BACKOFF, 1993) \\
\hline & Força da gestão operacional para ignorar o planejamento estratégico (JOYCE, 1999; MOTTA, 1998) \\
\hline \multirow{3}{*}{ HUMANA } & Liderança $(J O Y C E, 1999)$ \\
\hline & Comprometimento das pessoas (JOYCE, 1999; VILÀ; CANALES, 2008) \\
\hline & Envolvimento da alta administração (FLYNN; TALBOT, 1996; LOBATO, 2000) \\
\hline \multirow{2}{*}{ CULTURAL } & Visão legalista do orçamento (CUNHA, 1999) \\
\hline & Características culturais do brasileiro: improvisação, imediatismo e individualismo (LOBATO, 2000) \\
\hline \multirow{2}{*}{ POLITICA } & Definição de objetivos amplos e imprecisos (MOTTA, 2002) \\
\hline & Planejamento por mini análise (MOTTA, 2002) \\
\hline
\end{tabular}

$\checkmark$ Fonte: Elaborado pelos autores. 


\section{CONCLUSÕES}

Verifica-se que o investimento em técnicas e instrumentos de formulação de estratégias cada vez mais sofisticados, torna-se inócuo caso não haja a devida preocupação com a implementação dessas estratégias. Assim, deve-se estar atento a possíveis barreiras, que no setor público são muitas e intensas.

O planejamento estratégico não consiste apenas na confecção de um plano. Este se constitui em um processo, que requer que a estrutura organizacional esteja alinhada a ele, ou seja, os sistemas gerenciais são condicionados por ele.

Desta forma, percebe-se que o orçamento público não pode apenas servir como um instrumento de autorização de despesas. Ele deve se tornar uma ferramenta gerencial. Através da gestão orçamentária adequada, pode-se colocar o planejamento estratégico em prática.

Em geral, há inquietações em relação aos fracos ou, até mesmo, inexistentes laços entre os processos de planeja- mento estratégico e orçamentário na Administração Pública. Porém podem-se verificar alguns casos em que as organizações buscaram construir estes laços (GOODWIN; KLOOT, 1996; JOYCE, 1999).

Uma forma de se garantir que a estratégia influencie o processo orçamentário, e que os objetivos estratégicos serão contemplados é por meio da "orçamentação dupla"1. Esse mecanismo consiste na criação de um orçamento para iniciativas estratégicas separado do orçamento operacional. Tal mecanismo parte da suposição que a gestão operacional tem força suficiente para ignorar o planejamento estratégico. Desta forma, visa prover proteção às agendas estratégicas (JOYCE, 1999).

Tendo em vista que as especificidades do planejamento estratégico são pouco estudadas, além da relevância prática que tal assunto possui na gestão das organizações, sugerem-se pesquisas futuras que verifiquem como essas relações ocorrem empiricamente.

1 Tradução livre para dual budgeting.

\section{REFERÊNCIAS}

ABERNETHY, M. A.; BROWNELL, P. The role of budgets in organizations facing strategic change : an exploratory study. Accounting, Organizations and Society, v. 24, p. 189-204. 1999.

ANTHONY, R. N. Planning and Control Systems: A Framework for Analysis. Boston: Harvard University, 1965.

BARZELAY, M.; SHVETS, E. Improvisando as práticas de planejamento estratégico centrado em projetos. Revista de Administração Pública, v. 39, n. 3, 2005.

BERRY, F. S.; WECHSLER, B. Strategic planning: findings from a national survey. Public Administration Review, v. 55 , n. 2, p. 159-168, 1995.

BERTERO, C. O.; VASCONCELOS, F. C.; BINDER, M. P. Estratégia empresarial: a produção científica brasileira entre 1991 e 2002. Revista de Administração de Empresas, v. 43 n. 4, p. 48-62, 2003.

CUNHA, A. As Funções do Orçamento nas Organizações. Rio de Janeiro: EBAPE/FGV/CIPAD. , 1999

EADIE, D. C. Tool to Practical Use: Putting a Powerful of Strategic Planning The Application in the Public Sector. Public Administration Review, v. 43, n. 5, p. 447-452, 1983.

FERREIRA, V. R. S.; NAJBERG, E.; SOUSA, M. M. Pesquisa em Estratégia no Setor Público no Brasil: Avaliação da Produção Científica no Período 2007 - 2012. 2013, Bento Gonçalves:
ANPAD, Anais..., 2013. p. 1-11.

FLYNN, N.; TALBOT, C. Strategy and strategists in UK local government. Journal of Management Development, $v .15, n$. 2, p. 24-37, 1996.

GOODWIN, D. R.; KLOOT, L. Strategic communication, budgetary role ambiguity, and budgetary response attitude in local government. Financial Accountability \& Management, v. 12, n. 3, p. 191-204, 1996.

JOYCE, P. Strategic management for the public services. Buckingham: Open University Press, 1999.

LLEWELLYN, S.; TAPPIN, E. Strategy in the Public Sector: Management in the Wilderness. Journal of Management Studies, v. 40, n. 4, p. 955-982, 2003.

LOBATO, D. M. Administração Estratégica: Uma Visão Orientada para a Busca de Vantagens Competitivas. Rio de Janeiro: Editoração, 2000.

MIESING, P. Integrating planning with management. Long Range Planning, v. 17, n. 5, p. 118-124, out. 1984. Disponivel em: <http://linkinghub.elsevier.com/retrieve/ pii/0024630184900451>.

MINTZBERG, H. Ascensão e queda do planejamento estratégico. Porto Alegre: Bookman, 2004.

MOTTA, P. R. Gestão Contemporanea: A Ciência e a Arte de 
ser dirigente. 13. ed. Rio de Janeiro: Record, 2002.

MOTTA, P. R. Transformação organizacional: a teoria e a prática de inovar. Rio de Janeiro: Qualitymark, 1998.

NUTT, P.C.; BACKOFF, R. W. Transforming Public Organizations with Strategic Management and Strategic Leadership. Journal of Management, v. 19, n. 2, p. 299-347, 1 abr. 1993. Disponível em: <http://jom.sagepub.com/cgi/doi/1 0.1177/014920639301900206>

OSBORNE, D; GAEBLER, T. Reinventing Government: Introduction. Classics of Organization Theory. [S.I.]: Harcourt Brace, 1996.

PEARCE, J. A.; FREEMAN, E. B.; ROBINSON, R. B. The tenuous link between formal strategic planning and financial performance. Academy of Management Review, v. 12, n. 4, p. 658-675, 1987.
ROSE, W. R.; CRAY, D. Public-sector strategy formulation. Canadian Public Administration, v. 53, n. 4, p. 453-466, 2010.

SCHICK, A. Planing-Programming-Budgeting System: A Symposium. Public Administration Review, 1966.

SHIELDS, M.D. Research in management accounting by north americans in the 1990s. Journal of Management Accounting Research, v. 9, n. June, 1997.

STEWART, J. The meaning of strategy in the public sector. Australian Journal of Public Administration, v. 63, n. 4, p. 16-21, 2004.

VILÀ, J.; CANALES, J. I. Can Strategic Planning Make Strategy More Relevant and Build Commitment Over Time? The Case of RACC. Long Range Planning, v. 41, n. 3, p. 273-290, jun. 2008. Disponível em: <http://linkinghub.elsevier.com/ retrieve/pii/S0024630108000253>. Acesso em: 1 set. 2013.

\section{Como citar este documento:}

SOUZA, F. S. R. N. de; PARREIRA, T. J.; FONTENELLE, C. M. D. O Planejamento Estratégico como condicionante da Elaboração Orçamentária. Revista PAGMAR, Rio de Janeiro, v. 4, n. 4, p. 19 - 27, jan./dez. 2016.

\section{INOVAÇÃD, SEGURANÇA, AGILIDDEE E TRANSPRRENCIF.}

Reconhecida por seu pioneirismo na gestão de margem consignável, atendendo aproximadamente 2,5 milhões de servidores públicos por meio do eConsig , a Zetra amplia seu portfólio de soluções de tecnologia de ponta.

\section{ZOOM.RH}

Solução para o Gestor acompanhar e auxiliar o cumprimento da LRF (Lei de Responsabilidade Fiscal) na evolução das despesas da folha de pagamento.

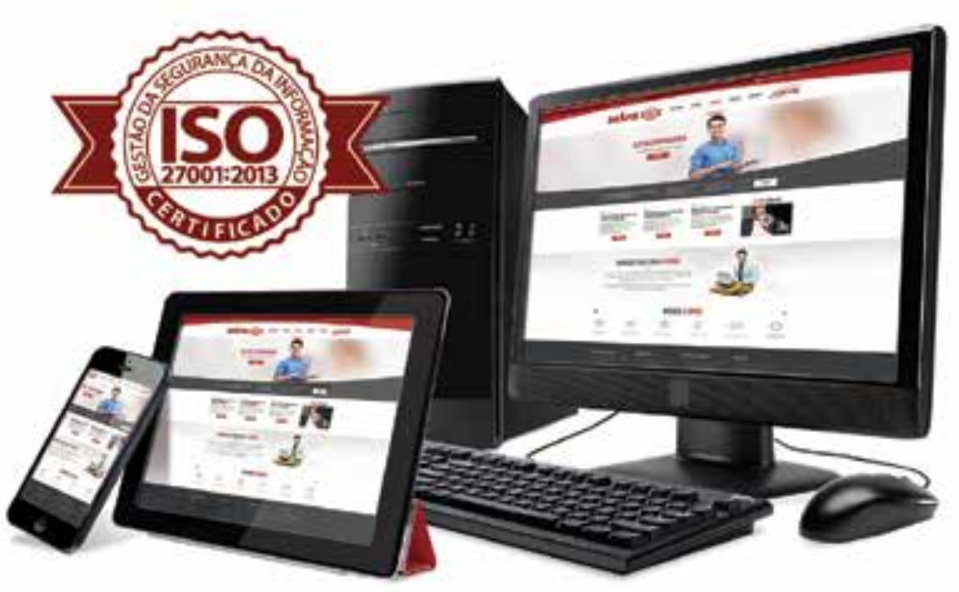

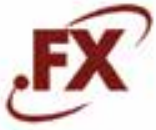

Utilizado pelas Consignatárias para produzirem propostas, contratos e formulários por meio de dispositivos móveis.

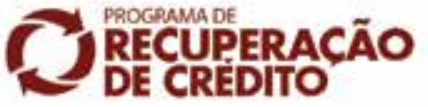

Voltado para os clientes do eConsig, tem como foco reduzir a inadimplência e o endividamento dos servidores públicos.

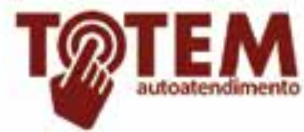

Permite consultar extratos, realizar simulações e demais serviços estabelecidos pelos gestores. 